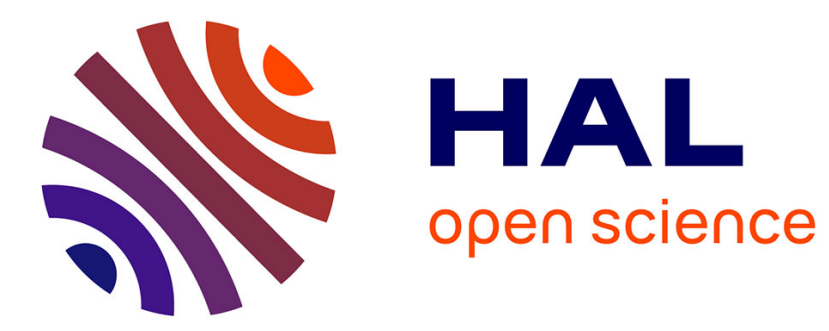

\title{
Art poétique et art de la guerre dans l'ancien monde touareg \\ Dominique Casajus
}

\section{To cite this version:}

Dominique Casajus. Art poétique et art de la guerre dans l'ancien monde touareg. L'Homme - Revue française d'anthropologie, 1998, 146, pp.143-164. halshs-00104588

\section{HAL Id: halshs-00104588 \\ https://shs.hal.science/halshs-00104588}

Submitted on 7 Oct 2006

HAL is a multi-disciplinary open access archive for the deposit and dissemination of scientific research documents, whether they are published or not. The documents may come from teaching and research institutions in France or abroad, or from public or private research centers.
L'archive ouverte pluridisciplinaire HAL, est destinée au dépôt et à la diffusion de documents scientifiques de niveau recherche, publiés ou non, émanant des établissements d'enseignement et de recherche français ou étrangers, des laboratoires publics ou privés. 


\section{Dominique Casajus}

\section{Art poétique et art de la guerre dans l'ancien monde touareg* Article paru dans L'Homme, 146, 1998 : 143-164.}

Le 28 novembre 1916, Charles de Foucauld écrivait dans son diaire : «Fini les poésies touarègues ». Composées entre 1820 et 1906, les poésies dont il achevait enfin, trois jours avant sa mort, la transcription et la traduction annotée constituent le seul document linguistique d'importance que nous aient laissé les Touaregs de l'époque précoloniale. Il avait recueilli cet ensemble de près de six mille vers, de février à juin 1907, auprès d'hommes appartenant aux trois confédérations les plus septentrionales du monde touareg : les Kel-Ähaggar, qui vivaient dans le massif de l'Ähaggar et l'Ädghagh malien et reconnaissaient la prééminence de la tribu noble des Kel-Ghela; les Täitoq et leurs tributaires, qui vivaient plutôt dans l'Ähnet, et sur lesquels les Kel-Ghela prétendaient exercer une suzeraineté souvent contestée ; les Kel-Äjjer, installés entre l'Ähaggar et la Tripolitaine, et où le rôle prééminent était joué par les Oûraghen.

Si l'hommage déférent à l'intérêt de ce corpus est devenu chez eux une figure obligée, les spécialistes du domaine l'ont dans l'ensemble ignoré, l'une des rares exceptions à ce désintérêt de fait étant constituée par les travaux de P. Galand-Pernet (1978, 1984) et L. Galand $(1980,1981,1986)$. Ma dette envers ces auteurs est ancienne, et le présent article doit en particulier beaucoup à la belle étude où L. Galand (1980) a montré tout ce que ces textes pouvaient nous apprendre sur l'art militaire dans l'ancienne société touarègue. Je voudrais les exploiter à mon tour pour tenter d'en dégager la vision que le guerrier touareg avait de son adversaire. En d'autres mots, je m'interrogerai sur le commerce qui liait ceux que la guerre opposait. Ce document me semble particulièrement adapté à une telle investigation car s'il existe des sources d'archives susceptibles de nous renseigner sur la pratique de la guerre chez les Touaregs, il est le seul à nous faire entendre leur voix et donc à pouvoir nous dire comment ils la vivaient ${ }^{1}$. Il nous montre en particulier que les relations guerrières étaient plongées dans le réseau continu de relations, empreintes de plus ou moins d'hostilité, qui innervait leur société. Je serai donc amené à quitter le domaine strictement guerrier et à parcourir tout au long ce réseau très emmêlé.

\section{La guerre intestine}

À l'examen de ce corpus, on remarque dès l'abord que, si les poètes chantent aussi bien la guerre intestine que la guerre étrangère, ils adoptent pour chacune d'elles un registre différent. Lorsqu'il est touareg, l'adversaire apparaît comme un proche, auquel on parle en même temps qu'on le combat; au contraire des Arabes ou des Français, dont nous verrons que les poèmes les confondent dans une masse 
indistincte et anonyme, on l'apostrophe en le nommant. Ces adresses peuvent prendre la forme de linvective: on en trouve un exemple dans un poème composé par Ähitaghel äg Biska lors de la longue guerre qui opposa les Kel-Ähaggar aux KelÄjjer de 1874 à 1878 (Voir Gardel, 1961 : 145 sqq.). Cette guerre avait eu pour origine les brimades que les Oûraghen faisaient subir à la tribu des Imenân, à la suprématie de laquelle leurs pères avaient mis fin au XVII ${ }^{\mathrm{e}}$ siècle. Les Imenân, dont il ne subsistait plus alors que quelques familles assez misérables, fuirent dans l'Ähaggar pour implorer la protection de l'ämenoûkal ${ }^{2}$ Elkhaj-Äkhmed. Après avoir tenté de négocier avec les Kel-Äjjer, celui-ci envoya contre eux une armée dont il confia le commandement à son cousin Ähitaghel äg Biska. La rencontre eut lieu en 1874 devant l'oasis de Ghât, et, après l'échec d'une ultime négociation, le combat aboutit à la défaite des Kel-Äjjer. Par la suite, ceux-ci prirent leur revanche au cours d'une bataille qui se déroula à Tañhart en 1875. Mais ils furent défaits sur le mont Oudjmîden en 1877, puis à I-n-ëleggi en 1878. On fit alors la paix. Le poème d'Ähitaghel célèbre le combat d'Oudjmîden, et l'auteur le conclut en interpellant Äkhnoukhen, l'ämenoûkal des Kel-Äjjer ${ }^{3}$ :

J'ai dit alors à Äkhnoukhen : "Approche, si tu l'oses, meure ta mère!

Je me suis vengé, malheur à ta mère!

Par Dieu, je te ferai fuir jusqu'à l'Äjjer de l'Est!»

On ne saurait affirmer que le chef kel-ghela a réellement harangué son adversaire sur le champ de bataille mais ce poème est en lui-même une apostrophe qui est vraisemblablement parvenue aux oreilles d'Äkhnoukhen. On sait d'ailleurs que les deux hommes ont échangé des plénipotentiaires alors que leurs armées campaient déjà face à face. Ce poème reprend donc un échange de paroles à peine interrompu par le combat ${ }^{4}$.

On trouve une allusion à une adresse semblable dans un poème composé avant que les Kel-Ähaggar et les Ioullemmeden de l'Äzaouagh se soient affrontés à Izerouân en $1896^{5}$ :

Ils ont envoyé dans l'Äzaouagh ce message :

«Dis à celui des Ioullemmeden qui est fils d'Ichiâd :

Attaquer par surprise est illicite, c'est un péché: [sache que nous t'attaquons]. »

Au contraire de la harangue d'Ähitaghel, ce message n'est probablement pas une invention de poète, car G. Alojaly, qui donne le point de vue des Ioullemmeden sur l'affaire d'Izerouân, parle lui aussi d'une lettre envoyée par les Kel-Ähaggar avant le combat (Alojaly, 1975 : 124). Et l'on a par ailleurs un autre exemple de cette pratique, puisque, dans une lettre au général Laperrine du 21 février 1915, C. de Foucauld rapporte que «... les Ioulliminden [sic] de l'Azaouar [sic], razziés il y a quelques mois une première fois par les Taïtoq et les Kel Rela [sic], une deuxième fois par Souri, Boukhelil et une bande rassemblés par eux, ont envoyé à Moussa 
[Moûsa ägg Ämâstan, alors ämenoûkal de l'Ähaggar] un message menaçant le prévenant qu'ils allaient venir l'attaquer » (Foucauld, $1954: 43$ ).

De même, en 1906, avant de partir en expédition pour mettre fin aux brigandages de Reskou äg Iakhia, Sîdi-Mokhammed äg Ghotman, des Kel-Ghela, composa à son intention un long poème comminatoire ${ }^{6}$. Reskou devait périr au cours de cette expédition : les défis, les invectives et les menaces dont les guerriers ornaient leurs poèmes ne restaient pas lettre morte.

Même quand ils ne s'adressent pas directement à eux, les poètes connaissent le nom de leurs adversaires, il les citent ${ }^{7}$, en particulier pour proclamer qu'ils les ont défaits $^{8}$ ou tués au combat. Ainsi, dans ce poème composé en 1899 à la suite de querelles entre Täitoq et Kel-Äir ${ }^{9}$ :

J'ai tué et laissé étendus sur le ventre Ämedderé et Äkhmâd,

j'ai fait d'eux la pâture des oiseaux ;

leurs mères sont dans le chagrin...

On lit de même dans un poème composé par Bâdi ägg Oughalla après le combat d'Oudjmîden ${ }^{10}$ : «Hemma et Äma sont déchiquetés et en morceaux étroits comme des bandes d'étoffe du Soudan. » Exagération poétique ? Que non point : les deux hommes eurent la tête, les mains et les pieds tranchés. Äma n'était rien moins que le fils d'Äkhnoukhen, et sans doute est-ce là la cause du traitement infligé à son cadavre par les vainqueurs. Alors même qu'on les massacrait cruellement, on connaissait donc ses adversaires, leur nom et leur parentèle. Pour ces hommes qui savaient tout les uns des autres, les batailles étaient plus une série de combats singuliers qu'une mêlée anonyme.

\section{Les duels poétiques}

À ces combats singuliers faisaient écho des duels poétiques, dans lesquels « de nombreuses pièces de vers, attaques et ripostes, [étaient] échangées » (Foucauld, 1925-1930, I : II). Ainsi, en 1875, peu avant le combat de Tañhart, Elghâlem ägg Ämedjoûr adressa au fils de l'ämenoûkal Elkhaj-Äkhmed ces paroles d'encouragement $^{11}$ :

Ghotman, abaisse ton voile sur tes yeux [toi qui vas] sur ta pouliche entraînée,

Nourrie de lait, rongeant son mors.

Commande en roi ce qui te plaît, nous te ferons une muraille de nos corps.

Les Kel-Ähaggar furent défaits, et Äkhenna ägg Ilbâk, des Kel-Äjjer, répondit en reprenant la même image, ce qui prouve que le poème d'Elghâlem lui était parvenu :

Elghâlem, la muraille que tu te vantais de faire a été mise en pièces [...]. 
Ghotman, tu l'as laissé mort sur la colline,

tu n'as pas tourné la tête vers lui quand il est tombé.

Deux ans plus tard, lorsque les Kel-Ähaggar prirent leur revanche à Oudjmîden, Elghâlem renvoya à Äkhenna une accusation similaire :

Äkhenna, ton cri de triomphe est retombé à terre,

toi qui, abandonnant Täghelift [homme inconnu], t'es enfui en courant.

Mais Äkhenna s'exclama alors :

Ils [les Kel-Ähaggar] ne nous ont vaincu que par ruse

et par une multitude égale à celle des mouches;

nous avions beau en chasser et en faire fuir jusqu'au sommet des collines,

leurs frères qui arrivaient derrière eux les ramenaient contre nous au combat.

Ce à quoi Elghâlem répliqua :

Ton dire a été reconnu faux, Äkhenna.

Où est la multitude par laquelle nous vous avons vaincus?

Notre victoire, c'est l'aide de Dieu qui nous l'a donnée.

À ces échanges résonnant de la fureur guerrière et du bruit des batailles, s'en ajoutaient d'autres, dont les auteurs embrassaient d'un regard plus large le cours du conflit et cherchaient à influer sur lui par une voie plus diplomatique. Ainsi, après le combat d'Oudjmîden, à un moment où des transactions avaient lieu entre Kel-Äjjer et Kel-Ghela, Äbekkeda äg Kelâla, des Oûraghen, entreprit de discréditer le parti de la paix $^{12}$ :

Je refuse absolument le lait qu'achèterait la paix

ainsi que l'or payé ce prix, y en eût-il une montagne,

tant que les femmes des Kel-Ghela n'auront pas eu une heure

chaude, en laquelle la fusillade pétille sur elles

et le soleil brûle leurs cheveux.

Éberzeouêl ägg Äzebbedah, des Kel-Ghela, lui répondit en ces termes :

En quoi la paix t'est-elle insupportable?

Toute paix qui se conclut sans contrainte et sans injustice est douce.

Les femmes des Kel-Ghela ont Dieu pour abri,

le lait de chamelle est leur nourriture,

c'est à l'ombre de l'épée qu'elles habitent.

Il existe des duels poétiques où la riposte reprend non seulement les images et les idées de l'attaque mais son mot à mot, ainsi qu'on le voit dans deux poèmes échangés en 1864, lors de démêlés entre les Kel-Ghela et les Täitoq. Doukka äg Khameidou, des Kel-Ghela, s'était écrié ${ }^{13}$ : 
Si je trouve des compagnons de rezzou, (Koud edjrëouegh täghara n ëmdâdj) Je les conduirai dans la vallée de Jîket faire une attaque qui sera pour elle comme des coups de cravache (Edjiq qen i Jîket ilekkâd)

sans même faire attention aux tentes de la plaine de Tälaq (Naki our edjrigh Tälaq fô dagh).

La même année, Sîdi ägg Äkeraji, chef des Täitoq, lui répondit :

Si je trouve des compagnons de rezzou, (Koud edjrëouegh täghara n ëmdâdj)

Je les conduirai dans la vallée de Tändjet faire une attaque qui sera pour elle comme des coups de cravache (Edjiq qen i Tändjet ilekkâd)

sans faire attention aux tentes du mont Äzîr-en-fad (Naki our edjrigh Äzîren-fad).

Hormis pour les noms de lieux-dits et pour la locution adverbiale fô dagh, supprimée dans le dernier vers, on voit que le second tercet est identique au premier. Cet exemple montre que les épigrammes guerrières étaient scrupuleusement transmises à leurs destinataires, et nous permet par la même occasion d'évaluer la fidélité de la mémoire orale. Songeons, en effet, que ces deux fragments ont été recueillis dans deux tribus différentes plus de quarante ans après leur composition. Or, il semble à peu près certain qu'ils se sont conservés fidèlement durant ce laps de temps, car si l'on supposait qu'ils se sont altérés, on ne pourrait comprendre par quel improbable hasard leurs déformations auraient été parallèles au point qu'ils soient restés mot pour mot la réplique l'un de l'autre ${ }^{14}$.

Bien qu'il ne s'agisse pas exactement de poèmes échangés par des adversaires, on peut citer un autre exemple de duel où les protagonistes reprennent dans leurs ripostes les termes mêmes de l'attaque. En 1906, tous les membres d'un rezzou parti vers le Sahara espagnol ayant péri de soif, à l'exception de trois hommes mûrs et d'un adolescent, Khelîfa, quelques personnes accusèrent les trois premiers d'avoir causé la mort de plusieurs de leurs compagnons. Nanna oult Äkadei, des Täitoq, se fit l'écho de ces accusations ${ }^{15}$ :

Dieu donne les biens et il désole (Mess-înegh ihâkken isârmâgh),

lui qui a réuni des hommes qui l'emportaient en tout, des nobles, pour les jeter dans le désert en pâture à la soif.

Il en a sauvé trois, auxquels est désormais interdit le violon ${ }^{16}$, et avec eux Khelîfa qui était en leur compagnie comme une âme en peine, il ne leur a manqué que de le balayer aussi.

Ätîsi äg Sedâda, des Ikadeien, et Mokhammed äg Ghëli, des Oûraghen, prirent chacun de son côté la défense des accusés. Le premier répliqua :

Nanna, Khelîfa a été ramené par des hommes au cour noble qui l'ont conduit jusqu'à sa mère Betâta, Violon!

Il ne leur reproche rien dans leur conduite envers lui ; [...] 
De tout temps les rezzous ont été tantôt malheureux, tantôt heureux. (Idjnân iroû ghâssheden kânnen)

Si ces hommes ne sont pas morts, c'est qu'ils n'avaient pas achevé le temps de vie que Dieu leur a prédestiné : (Innîn our emman, our essemden)

C'est Dieu qui a refusé de les laisser mourir... (Mess-înegh a ioudjëien ser sen)

et le second :

Les rezzous, de tout temps, ont été remis à plus tard ou se sont faits ; (Idjnân iroû tâmeouin tâdjdjen)

ceux dont le temps de vie prédestiné par Dieu n'était pas achevé en sont revenus saufs; (Éré s our emdin hadân islëm)

c'est Dieu qui donne les biens (Mess-înegh a itâdjdjen errezghen),

c'est lui qui fait mourir celui qui vit dans l'abondance et le bonheur.

Les trois auteurs ont recours à la tournure "Mess-înegh (Dieu) + participe », ce participe étant à l'aoriste intensif dans deux cas sur trois. Plus net encore, Ätîsi et Mokhammed utilisent tous deux la construction «idjnân iroû (les rezzous depuis longtemps) + deux aoristes intensifs à la 3ème personne du pluriel ». On peut ajouter l'utilisation qu'ils font tous deux du verbe emdou au sens assez inhabituel de «s'achever du fait de la volonté de Dieu ». Sans l'être autant que dans l'exemple précédent, nous sommes encore assez proches de la répétition mot à mot, alors pourtant que les trois auteurs appartiennent à trois confédérations différentes.

\section{Les femmes et les guerriers}

Après les échanges poétiques entre ennemis, dont C. de Foucauld a pu justement écrire: "[1]es guerres sont presque toujours accompagnées d'une lutte poétique » (Foucauld, 1925-1930, I : II), nous allons maintenant considérer les poèmes que les belligérants adressaient à leur propre camp. Un peu comme dans les textes destinés aux adversaires, leurs auteurs protestent de leur bravoure et évoquent la conduite de leurs compagnons, louant la vaillance des uns et flétrissant la couardise des autres ${ }^{17}$. On ne retrouve guère cependant de joutes poétiques semblables à celles dont le paragraphe précédent a fourni des exemples. Plutôt qu'à d'autres poètes, les guerriers répondent ici à ce bruissement confus de paroles indistinctes qu'on appelle la rumeur ${ }^{18}$. Compagne des batailles, c'est elle qui décide de la gloire ou de l'opprobre des combattants, les poètes la sollicitent, l'orientent, la font enfler, ou s'en défendent. Ainsi, en 1865, un homme des Kel-Ähaggar ayant participé l'année précédente à un combat contre les Täitoq, se défend des accusations de lâcheté qui courent sur son compte et dit son espoir que des bruits plus favorables finiront par les balayer ${ }^{19}$ :

Si j'ai abandonné mes armes ou mes amis où que ce soit, s'il existe des hommes et une vérité, 
cela se saura, mes compagnons ne le cèleront pas.

C'est aux femmes que les poètes se soucient particulièrement que la rumeur parvienne. Ils leur dédient leurs poèmes, ils les invoquent ${ }^{20}$, ils les prennent à témoin de leur bravoure ou de la lâcheté de leurs compagnons ; on le voit, par exemple, dans ce poème composé après le combat d'Izerouân ${ }^{21}$ :

Je tins mon bouclier devant mon visage, j'allai à l'ennemi.

Les javelots tombèrent sur nous

comme une pluie d'orage accompagnée de tonnerre [...]

Dites-le à celles qui parlent en adoucissant les sons,

à celles qui sont dans la vallée de Tesâmeq vers les gorges

et à celle dont l'amour ne sortira pas de mon cour

tant que ne pèseront pas sur moi les pierres du tombeau.

Ou dans ce poème composé en 1895 après un combat contre les Ioullemmeden $^{22}$ :

J'envoie à toutes les femmes qui prennent part aux réunions galantes un arrêt,

à celles de ce pays-ci et même à celles des contrées arabes :

en quelque lieu que se tiennent près de vous ceux qui se sont cachés pendant ce combat,

répandez sur eux vos malédictions.

Mêlant la cruauté à la galanterie, on semble parfois mettre à leurs pieds les dépouilles des vaincus, comme on le voit dans le poème déjà cité de Bâdi ägg Oughalla. S'adressant à une dame souvent invoquée par les poètes ${ }^{23}$, qui «tenait le premier rang parmi les femmes des Imenân, par sa naissance et par sa beauté » (Foucauld, 1925-1930, I : 123), l'auteur s'écrie : « Tu as été vengée, Täkharibt, en un pays lointain $»^{24}$. Puis il décrit, gage de l'accomplissement de cette vengeance, le supplice infligé au fils d'Äkhnoukhen, avant d'énumérer ce qu'il lui a «pris sur le champ de bataille $»^{25}$ :

... le méhari brun-rouge d'Äma et ses riches vêtements,

son fusil, son épée, son javelot,

et sa grande calotte rouge près de laquelle passaient les baudriers.

Il arrive que les guerriers s'adressent aux femmes du camp adverse ; parfois pour les moquer, tel Doukka äg Khameidou se riant, après le combat de Ghât, de celle dont il a razzié le méhari ${ }^{26}$; ou Enbâra ägg Oûragh faisant mine, dans un texte datant de 1904, d'interpeller galamment les femmes dont il vient de razzier les campements $^{27}$ : «Les femmes des Kel-Fedai, dites-leur: "Ne fuyez pas, écoutezmoi, /nous venons vous faire des visites galantes et assister à vos ähâl $l^{28}$, notre présence les rendra brillants." ». 
D'autres recherchent plutôt leurs suffrages. Ainsi, en 1892, un homme des Kel-Äjjer allant en ambassade chez les Kel-Ghela pour leur réclamer des troupeaux razziés aux siens, apostrophe les femmes Kel-Ghela en flétrissant à leurs yeux l'inconduite de leurs propres contribules ${ }^{29}$. Dans le même ordre d'idée, on peut citer un poème où, en 1905, après une querelle avec des Täitoq, un jeune Kel-Ghela rappelle la lâcheté d'un de ses adversaires en s'écriant: «Il a oublié Kherîba aux beaux colliers ${ }^{30}$. Cette Kherîba que l'auteur connaît par son nom est une Täitoq, de sorte qu'il faut penser qu'il se sentait assez proche de ses adversaires et de leurs compagnes pour prendre plaisir à déconsidérer les premiers aux yeux des secondes. On a vraiment là l'impression d'une guerre entre proches, où les adversaires disputaient l'admiration des mêmes filles. En un sens, femmes amies et femmes de l'ennemi sont traitées ici de semblable manière. Il n'en était pas toujours ainsi dans la réalité ; s'il est vrai, par exemple, qu'Ähitaghel traita humainement les épouses et les sœurs des vaincus, venues implorer sa pitié après le combat d'Oudjmîden ${ }^{31}$, on sait aussi que les femmes étaient parfois maltraitées lors des razzias $^{32}$; mais il n'en demeure pas moins que les poètes se souciaient de ce que le bruit de leur gloire parvînt jusqu'aux oreilles des compagnes de leurs ennemis.

Cette présence des femmes dans la poésie guerrière, en témoins et juges de la valeur des guerriers, rattache l'un à l'autre un genre guerrier et un genre élégiaque dont L. Galand a bien remarqué que sans cesse ils s'entremêlent (Galand, 1980 : 109111 ; voir aussi P. Galand-Pernet, 1978 et 1984). Comme l'a relevé cet auteur, que je vais suivre ici, le guerrier se montre en chemin vers le lieu du combat, aussi richement habillé et presque dans les mêmes dispositions d'esprit que s'il se rendait à l'ähâl. Dans un poème composé lors de la guerre entre Kel-Ähaggar et Kel-Äjjer, l'auteur compare même la bataille à venir à une réunion galante ${ }^{33}$. Un autre, en 1880 , confond dans la même louange la bravoure de ses compagnons et leur élégance devant les dames $^{34}$ :

Je fais compagnie avec des jeunes gens dont tous disent les louanges ;

depuis leur naissance, ils ne savent que faire des pointes hardies au milieu des ennemis,

aller à la réunion galante au coucher du soleil, et marcher en approchant des femmes d'un pas lent et solennel.

Citons de même un court et beau poème datant du 1870, où se confondent admirablement la galanterie, la passion guerrière et même une piété rarement présente dans ces poèmes ${ }^{35}$ :

Très-Haut, je tends mes mains vers toi ;

je te fais cent et mille prières ;

Très-Haut, je te demande trois choses :

l'amour des jeunes filles, la vaillance dans les combats, et le pardon le jour de la résurrection. 
Souvent, l'évocation des combats n'est que le prétexte à un développement élégiaque, où le poète, songeant à celles qu'il a quittées et qu'il retrouvera chargé d'honneurs ou couvert de honte, dit dans des termes qui sont ceux de la poésie amoureuse sa souffrance d'être séparé d'elles ${ }^{36}$. Comme dans ce poème que Moûsa ägg Ämâstan composa en 1896, après un rezzou contre les Ioullemmeden ${ }^{37}$ :

Mon cour a été blessé quand j'ai quitté celle qu'aime mon âme. [...]

Je me suis roulé par terre sans pouvoir me résigner à son absence dans les lieux boisés de grands arbres, perdu dans sa pensée.

J'ai marché à travers des dos sablonneux couverts de végétation et des dunes

portant mon tourment et excitant du pied mon méhari dans la force de l'âge.

Je me suis jeté dans les risques, non sans forces suffisantes.

J'ai pris pour objectif un fort groupement d'Ioullemmeden...

À l'inverse, L. Galand le souligne là encore, il est des poèmes galants où la guerre est métaphoriquement présente. Les femmes aimées y sont dépeintes comme aussi cruelles que les guerriers d'un rezzou ennemi, telle cette Helba oult Ebbah, dont la présence en image à l'esprit du poète est plus mortelle pour lui «qu'une livre de poudre, plus mortelle que les javelots / enfoncés dans l'abdomen et qu'on en retire en arrachant les entrailles », ou telle l'inconnue qui inspire un amour «plus brûlant que de la terre sur laquelle est un brasier, plus brûlant / que les balles des Turcs reçues en plein front $»^{38}$.

\section{La guerre pour un air de violon}

Un thème récurrent illustre particulièrement la présence de la galanterie dans la poésie guerrière et dans la guerre elle-même : celui du violon (Galand, 1980). Les dames en effet jouaient du violon en l'honneur des partants lors du départ des rezzous $^{39}$ et honoraient de même les preux revenus du combat. Et l'on voit des poétesses promettre ces airs de violon à des hommes valeureux et aimés ${ }^{40}$. La pensée du violon qui attend à leur retour les plus vaillants d'entre eux accompagne les guerriers lorsqu'ils partent au combat. Ainsi dans ce texte datant de $1877^{41}$ :

Le rezzou part: je prépare mes vivres :

ce sont l'air du «dernier tiers de la nuit » et celui des « ikedaouâten » [noms d'air de violon],

Tabhaout tient le violon sur ses genoux.

L'homme à qui ces airs ne plaisent pas, qu'il sorte

d'entre les guerriers du rezzou, quand ceux-ci partiront et iront en aval. 
Au lâche, on annonce au contraire qu'il en sera à jamais privé. On l'a vu plus haut dans le poème de Nanna oult Äkadei. Un autre est ironiquement accusé d'avoir oublié « le violon qui joue / chez la fille d'Ahar qui sourit en montrant ses dents ${ }^{42}$; un autre encore s'entend dire, en $1865^{43}$ :

Retourne aux réunions galantes, entre les joueuses de violon et leurs compagnes, si tu l'oses, et rougis de honte!

La nouvelle de ta conduite va parvenir à la femme aux dents blanches

et à toutes les jeunes femmes qui sourient et se polissent le visage.

Et l'expression «violon ! ", déjà rencontrée dans un des extraits cités plus haut, finit par devenir une exclamation guerrière ${ }^{44}$.

Il est arrivé une fois que ce violon au son duquel était célébrée la gloire des combattants devînt lui-même l'objet d'affrontements guerriers. «Le jour du combat de Tañhart, en pillant les tentes des Kel-Ghela, les Kel-Äjjer enlevèrent un beau et grand violon fort connu, qui appartenait à Tèhît oult Hedjîer, l'une des plus belles parmi les jeunes femmes nobles de l'Ähaggar. » (Foucauld, 1925-1930, I : 125) Ils l'offrirent à une de leurs dames, elle aussi grande joueuse de violon ${ }^{45}$. Après le combat d'Oudjmînen, Bâdi äg Oughalla le retrouva parmi les dépouilles des Kel-Äjjer. Dans son poème déjà cité, il se décrit ainsi au retour du combat ${ }^{46}$ :

Je reviens, faisant marcher mon méhari acajou clair d'un pas lent et cadencé,

tenant sur mes genoux le violon

reconquis par la force du bras.

Saluez de ma part Täkharibt et toutes les joueuses de violon!

Il faut que ce violon ait été bien précieux, et sa belle propriétaire particulièrement chérie des guerriers, puisque Bâdi le tient pour un butin d'aussi grand prix que les dépouilles arrachées au cadavre d'Äma, à l'énumération desquelles il a consacré les vers précédents ${ }^{47}$. La galanterie, on le voit, colorait parfois la guerre elle-même et non plus seulement les poèmes qui la chantaient.

\section{Rivalité guerrière et rivalité amoureuse}

De même que le genre guerrier puisait son inspiration aux mêmes sources que le genre élégiaque, de même la rivalité guerrière se nourrissait quelquefois de la rivalité amoureuse. Ainsi, Beika äg Moummou, dont Mokhammed äg Mekhiia dénonce la lâcheté dans plusieurs poèmes et dont il fait souvent la cible de ses épigrammes $^{48}$, avait épousé une femme que tous deux aimaient. De façon comparable, plusieurs poèmes ${ }^{49}$ évoquent une rixe au cours de laquelle des adolescents Kel-Ghela et Täitoq échangèrent quelques coups de fusils, et que seule l'intervention rapide des adultes empêcha d'être meurtrière. «On se réconcilia bientôt, car les jeunes Kel-Ghela aimaient les réunions galantes des femmes Täitoq, les jeunes 
Täitoq aimaient celles des femmes Kel-Ghela, et la brouille entravait les plaisirs habituels. » (Foucauld, 1925-1930, I : 571). Voilà donc des jeunes gens qui courtisaient les mêmes filles et qui se seraient entretués si leur jeunesse n'avait limité les effets de leur querelle. Le rival en amour pouvait donc, à l'occasion, devenir le rival dans le pourchas de la gloire, et il s'en fallait parfois de peu qu'il ne devînt l'ennemi. L'affinité entre genre guerrier et genre galant tendait donc à déborder le domaine de la seule poésie, et à se doubler d'une certaine affinité entre l'attitude face à la guerre et l'attitude face à la conquête amoureuse.

Le thème de la rivalité amoureuse peut également apparaître dans des poèmes dont toute inspiration guerrière est absente ${ }^{50}$. On le rencontre encore dans la poésie contemporaine, où il a survécu à un genre guerrier dont il est peut-être un lointain souvenir. Semblable au poète épique de jadis, qui s'affirmait plus brave, et partant plus digne d'amour, que des ennemis ou des compagnons d'armes devenant dans ses vers des rivaux en amour, le poète élégiaque d'aujourd'hui prend à témoin des interlocutrices imaginaires de ce qu'il surpasse dans l'art poétique ses rivaux en amour et ne les craint pas au combat (Voir Prasse, 1990 : 13 s. ; Albaka et Casajus, 1992, passim). L'affinité entre les deux genres est donc assez sensible pour que, l'un disparu, l'autre en fasse encore résonner l'écho.

Autre signe de cette affinité, la rivalité amoureuse suscitait des dialogues poétiques comparables à ceux que produisait la guerre ${ }^{51}$. Khamîd ägg Äfîser et Elghâlem ägg Ämedjoûr n'ont cessé d'entretenir une rivalité poétique qui semble d'ailleurs être restée amicale, même lorsqu'elle s'est doublée de la rivalité amoureuse. Ils se livrèrent un jour, dit-on, à une joute poétique dans la tente d'Ähitaghel äg Biska, alors devenu ämenoûkal (Foucauld, 1925-1930; I : 45). De telles joutes n'étaient peut-être pas exceptionnelles, comme en témoignent également les quatre poèmes improvisés par quatre épouses successives de Sîdi äg Äkeraji, un jour qu'elles voyageaient de conserve ${ }^{52}$.

Variante du poème inspiré par la rivalité amoureuse, un genre bien représenté est celui où l'auteur déplore le mariage de celle qu'il aime avec un tiers, tantôt feignant le dédain, tantôt confessant sa peine ${ }^{53}$. Prenons l'exemple d'un poème de Moûsa ägg Ämâstan datant de 1891, qui passe successivement par les deux mouvements. Après avoir dénigré la « misérable petite tente » où une femme qu'il aimait a pris époux, ce qui est coutumier dans ce type de poème, l'auteur laisse éclater sa douleur et ses regrets $^{54}$ :

Maintenant, je le déclarerai franchement [...]

Je t'aime, Tehôr-d-eddel, je t'aime de plus en plus, ton amour attise un feu dans mon âme,

cesser de t'aimer, quelque effort que je fasse, est aussi loin de moi que les étoiles.

Jamais un homme ne me fera jurer de renoncer à elle, 
fût-il un lion paraissant devant moi...

Ailleurs, un homme qui a épousé une femme admirée de tous, se vante au contraire d'avoir mis ses rivaux en fureur, ce qui lui vaut une réplique dont la sécheresse n'a rien à envier à celle des poèmes guerriers ${ }^{55}$.

\section{Une vie sociale tissée de poèmes}

Toute la vie galante, et finalement toute la vie sociale, étaient tissées de tels poèmes. Le thème de la réunion galante, déjà présent dans les poèmes guerriers notamment sous la forme des allusions au violon, y est souvent développé ${ }^{56}$. Des dames repoussaient sans ménagement des galants maladroits, dont d'autres prenaient la défense ${ }^{57}$. On marivaudait parfois en vers, comme cette Ta-nnes oult Melloül, qui, en 1903, fit reproche à Äkhou Äg Beika de l'avoir abordée en lui disant «ma-nëouîn? », formule de salutation sans signification particulière, et non «isalân ? », le salut galant d'un homme à une femme ${ }^{58}$. Ce à quoi Äkhou répondit ${ }^{59}$ :

Ta-nnes, laisse les paroles sans raison, où veux-tu en venir?

L'autre soir, quand je suis allé te voir au coucher du soleil, tu savais bien que c'est pour une conversation galante que j'allais chez toi.

Et toi, n'as-tu pas fait autre chose que me dire «comment vas-tu» (ma-nëouîn)?

Tu trouves que je suis un vieillard?

Les accidents de la vie conjugale donnaient lieu à des poèmes, où se diffusaient les rumeurs courant sur l'infidélité de l'un ou de l'autre, et auxquels les accusés répondaient avec acrimonie ${ }^{60}$. Les épouses s'y montraient volontiers insolentes, comme cette femme disant sa joie d'avoir pu fuir un Arabe à laquelle on l'avait mariée peut-être contre son gré, ou cette autre rendant public un amour coupable afin d'être répudiée ${ }^{61}$. Citons également dans cette veine la réplique de Lalla oult Illi à son mari Etteiioûb, qui, vers 1895, lui reprochait d'être trop accessible aux avances de Moûsa ägg Ämâstan ${ }^{62}$ :

Dis à Etteiioûb : meure ton père! écoute bien :

Moûsa ägg Ämâstan n'a pas son pareil ;

homme tout jeune, il est fait pour les baisers.

La place qu'il voudra dans mon cour il l'aura.

C'est Lalla fille d'Illi qui a dit cela.

On trouve aussi, et là nous sortons de la galanterie, des poèmes qui sont des conseils, tels ceux donnés par des poétesses à des hommes repoussés par celles qu'ils aiment. D'autres sont des remontrances à des personnes ayant agi contre les usages : une femme reproche à son frère de trop tarder à conduire son épouse dans son campement; un jeune homme blâme ses compagnons d'avoir mangé un méhari, monture habituelle d'une jeune fille ; un homme fait honte à une femme qui a mangé 
un de ses méharis ; une jeune fille gémit d'avoir été durement traitée par des amis tendrement aimés ; une femme déplore qu'on ait manqué de courtoisie à la réunion galante ; une autre s'indigne de ce qu'un homme laisse son père mourir de faim ; une autre encore se plaint de ce que ses amis ne fréquentent plus guère les réunions galantes qu'elle donne, une autre enfin qu'on ait manqué à son égard aux devoirs de l'hospitalité, etc. ${ }^{63}$

Certains poèmes sont des injures ou des menaces échangées au cours de querelles $^{64}$. D'autres enfin sont de simples plaisanteries, tels ces poèmes où soit un homme taquine les femmes de sa tribu en louant celles d'une tribu voisine, soit une femme fait de même pour taquiner les hommes de sa tribu ${ }^{65}$. Il est des cas où ces plaisanteries sont si grossières qu'on les soupçonne d'avoir été échangées entre cousins croisés $^{66}$. Dans toutes ces épigrammes, le ton reste le même, qu'elles soient échangées entre contribules ou entre membres de deux tribus différentes ; des parents s'invectivent parfois aussi violemment que le font les ennemis en guerre ${ }^{67}$.

\section{De la civilité de la guerre}

Avant de passer à l'examen des poésies consacrées à la guerre étrangère, il est possible de tirer un premier bilan de cette étude. Les textes examinés jusqu'ici forment un spectre continu où se disposeraient successivement, par ordre d'hostilité croissante, la pièce de circonstance où l'on moque ou morigène l'ami proche et affectionné, le trait tantôt plaisant tantôt acrimonieux décoché au rival amoureux, puis l'épigramme lancée au compagnon d'armes aux côtés de qui l'on pourchasse la gloire, et enfin les poèmes véhéments transmis à l'ennemi entre deux batailles.

Cela donne à la guerre, lorsqu'elle déchire les Touaregs, tous les caractères de la guerre civile. Qu'on ne se méprenne pas sur ce mot. Je n'affirme en rien que nous serions en présence d'une entité politique bien délimitée à l'intérieur de laquelle une guerre se définissant comme civile s'opposerait à la guerre étrangère. C'est, à l'inverse, la civilité dont ces poèmes imprègnent la guerre intestine qui autorise à parler d'une cité touarègue, ou pour employer un terme aux connotations moins lourdes, d'un monde touareg - au sens où Jean-Pierre Vernant évoque « un monde grec qui, dans leur affrontement même, rassemble les cités en une communauté unie par la langue, la religion, les mœurs, les formes de vie sociale, les modes de pensée » (Vernant, 1968 : $20)^{68}$. Dans ces poèmes en effet, mon adversaire est un proche que j'appelle par son nom. Les termes dans lesquels je l'apostrophe ne diffèrent qu'en degré de ceux dont j'use lorsque je converse poétiquement avec mes contribules, et les images dont j'orne alors mon propos sont souvent semblables à celles dont je nourris les poèmes que je destine aux miens. Je sais qu'il partage le même éthos que moi et que les actes dont je m'enorgueillis sont ceux-là mêmes dont il se fait gloire lorsqu'il les commet. C'est pourquoi, en même temps que je lui porte mes coups, j'ai à cœur que lui parviennent des poèmes où je les célèbre, et il me répond à son tour mot pour mot tandis qu'il me 
rend coup pour coup. D'ailleurs, les femmes que je prends si volontiers à témoin de ma vaillance sont aussi bien les siennes que mes propres amies, tant je suis sûr que de son côté comme du mien, les canons de la valeur sont les mêmes. À ce semblable, ce frère contre qui toujours je guerroie, me lient de secrètes connivences, que nos poèmes indéfiniment échangés ne cessent de trahir.

Certes, l'ennemi, même touareg, était l'ennemi. Ainsi, nous l'avons vu, le poème dans lequel Bâdi ägg Oughalla conte avec préciosité l'épisode du violon arraché aux Kel-Äjjer est aussi celui où il jette aux pieds de la belle Täkharibt le cadavre mutilé du fils d'Äkhnoukhen. Car ces guerres entre proches étaient cruelles et l'on s'y combattait sans merci. On le perçoit également dans un poème mettant en scène un Moûsa ägg Ämâstan hébété de souffrance après la mort de son frère et déterminé à le venger chèrement, ce qu'il devait faire lors du combat d'Izerouân ${ }^{69}$. On pourrait croire à quelque artifice littéraire dans cette mise en scène d'un Moûsa peint en Achille pleurant la mort de Patrocle et aspirant comme lui à la vengeance. Mais d'autres documents, tels les textes en prose recueillis en 1906 par C. de Foucauld et A. de Cassalanti-Motylinski (Foucauld et Cassalanti-Motylinski, 1984 : 24), ainsi que la chronique de G. Alojaly (Alojaly, 1975 : 120-132), attestent la réalité de la fureur de Moûsa et la persistance de la haine entre Kel-Ähaggar et Ioullemmeden. G. Alojaly montre d'ailleurs combien les traîtrises et les actes de cruauté étaient fréquents dans les guerres que se livraient les Touaregs. La haine qui pouvait s'y donner libre cours, et dont on a vu un exemple avec le sort cruel fait au fils d'Äkhnoukhen, était ellemême le signe de la proximité que se reconnaissaient les adversaires : on ne hait personne autant que celui dont on connaît le nom et la famille, à qui on peut dire sa haine dans des mots qu'il comprend, et qu'on peut frapper dans des affections dont on sait combien elles lui sont précieuses.

\section{La guerre étrangère}

J'ai anticipé dans ce qui précède sur l'examen encore à faire du traitement que les poètes réservent à la guerre étrangère, car il va de soi que le bilan partiel qui vient d'être dressé suppose implicitement que ce traitement diffère significativement de celui qu'ils accordent à la guerre intestine. Nous allons voir que c'est, en effet, le cas. La guerre étrangère que célèbrent ces poèmes est tout d'abord celle qu'on faisait aux tribus arabes circonvoisines. Entre celles-ci et le monde touareg, la frontière n'était ni étanche ni clairement définie, et les hostilités intermittentes n'excluaient pas les alliances temporaires dans des conflits où chacun des deux camps voyait se mêler Arabes et Touaregs. En 1860, quelques Kel-Ähaggar firent avec les Oulad-BaHammou une razzia sur les Oulad-Essaieh ; en 1875, après leur défaite devant Ghât, les Kel-Äjjer s'unirent à des Arabes du Fezzan pour prendre leur revanche contre les Kel-Ähaggar; en 1891, les Täitoq et les Kel-Ähnet s'unirent aux Kounta dans une expédition punitive contre les Oulad-Moulât; en 1899, les Täitoq appelèrent à leur 
secours des Arabes du Tidikelt lorsque des démêlés les opposèrent aux Kel-Ghela ${ }^{70}$. Il arrivait qu'une tribu arabe recherche la protection d'un chef touareg, lui fasse allégeance et finisse même par être assimilée. La tribu touarègue des Kel-Ghezzi, de même que les Iseqqemâren, qui constituaient un ordre social intermédiaire entre les nobles et les roturiers, passaient pour être d'origine arabe (Nicolaisen, 1963 : 398). Des Arabes se sont installés en pays touareg et y ont fait souche: des deux principaux collaborateurs de C. de Foucauld, l'un, Ba-Hammou, était arabe, et l'autre, Mokhammed äg Ghëli, était de père arabe.

Pourtant, si floue et si perméable qu'elle ait été, nos poèmes font bien apparaitre qu'une invisible frontière séparait les Touaregs de leurs voisins arabes. Il pouvait sans doute arriver que ceux-ci soient invectivés comme des adversaires touaregs. On le voit dans le texte suivant, non daté par Foucauld ${ }^{71}$ :

Les Arabes, la respiration est également en nous et en eux :

s'ils marchent contre nous sur leurs juments noires,

décrivant au galop des cercles autour de nos guerriers, je tiens mon long fusil à pierre, mon cour n'est pas lâche, certains ne passeront pas longtemps sans tomber.

Mais on ne s'adressait jamais nommément à l'un d'eux. La seule exception à ma connaissance se trouve dans un poème recueilli en 1858 par A. Hanoteau, qui commence par le distique : «Meurs ton père, Matalla ! Es-tu possédé du démon ?/ Crois-tu donc que les Ifor'as ne sont pas des mâles ?» (Hanoteau, 1896 : 211 ; voir une version voisine dans Duveyrier, 1864 : 450-451). On peut citer aussi un cas où le nom d'un chef arabe est mentionné ${ }^{72}$. Mais tout cela est bien trop peu pour qu'on puisse nier que la guerre avec les Arabes se montre dans la poésie dépourvue de cette connivence qui relie les Touaregs quand même ils se combattent. Et on n'y trouve de toute façon aucun de ces duels poétiques auxquels donnent lieu les guerres intestines. Alors que dans la guerre entre Touaregs, les destinataires des poèmes sont ceux avec qui on croise le fer, les dialogues poétiques restent ici internes au monde touareg. Lorsqu'on s'adresse à quelqu'un, ce n'est pas à l'ennemi arabe mais au compagnon d'armes touareg à qui l'on reproche son manque de courage ${ }^{73}$, aux contribules devant qui on se défend d'une accusation de lâcheté ${ }^{74}$ ou aux dames qu'on invoque et à qui l'on dit sa bravoure ${ }^{75}$. La razzia désastreuse contre les Regîbât du Sahara espagnol dont j'ai parlé plus haut a surtout donné lieu à des accusations réciproques entre Touaregs, et dont les Regîbât eux-mêmes sont presque absents ${ }^{76}$.

On trouve les mêmes traits, encore plus accusés, dans les poèmes inspirés par les conflits avec les Français. Le combat de Tit, qui vit en 1902 plus d'une centaine de Kel-Ähaggar fauchés par les fusils à tir rapide des tirailleurs chaamba conduits par le lieutenant Cottenest, a inspiré un grand nombre de poèmes. Le nom de Cottenest est certes mentionné dans l'un d'eux ${ }^{77}$ mais le cas est particulier puisque l'auteur, Mokhammed äg Ghëli, a combattu du côté français et servi de guide à Cottenest. 
Pour le reste, les invectives, même collectives, sont totalement absentes. Tous les poèmes s'adressent à des Touaregs, hommes ou femmes, dans des termes semblables à ceux qu'inspirent les guerres intestines ${ }^{78}$.

Un autre trait apparaît dans les poèmes célébrant la guerre contre les Français. Ceux-ci sont appelés les «païens $»^{79}$, et ce, même par des poètes qui ne leur sont pas hostiles, comme Mokhammed äg Ghëli, ou comme telle vieille femme ayant composé un poème en l'honneur d'un officier qui lui avait fait une aumône ${ }^{80}$. Ceux qui se soumettent à eux sont des apostats ${ }^{81}$. La guerre qu'on leur fait est sainte, comme le montre, par exemple, le poème suivant, composé en $1905^{82}$ :

Ô vous, au nom de Dieu et pour l'amour des saints, où que vous soyez, avant tout, marchez contre eux; quoi qu'il arrive, ils n'auront pas l'aide de Dieu : ils marchent dans la désobéissance au Prophète, ils sont destinés à un grand feu dans l'enfer.

Mieux vaut pour vous gagner, par la guerre sainte, les récompenses célestes que de vous soumettre à des hommes à bouche non voilée et à moustaches de chiens.

Ce poème a une tonalité religieuse qu'on ne trouve presque jamais dans les poèmes composés lors des guerres intestines. Les vers, fort désobligeants à l'égard des Français, par lesquels il s'achève, sont exceptionnels : en général, dans ce genre de poème, l'accusation d'impiété est dirigée moins contre les Français eux-mêmes, dont on ne semble guère se soucier directement, que contre ceux qui ont pris leur parti, se chargeant ainsi du péché d'apostasie. Dans un poème datant de 1903, dont l'auteur reproche longuement à l'un des siens de s'être soumis aux Français, l'accusation est même doublement interne au monde touareg. Elle l'est tout d'abord en ce qu'un Touareg s'y adresse à son contribule mais elle l'est aussi parce que, dans la manière de sermon qu'il compose à son intention, les biens terrestres auxquels il lui reproche d'être attaché au point d'en avoir oublié les châtiments que l'au-delà réserve à l'apostat, sont ceux-là même que tous ces poèmes n'ont cessé de chanter, et à la poursuite desquels les Touaregs épuisaient leur jeunesse ${ }^{83}$ :

Boûsîf, ton pays a apostasié :

le Commandant [Laperrine] y est allé et l'a soumis, lui qui ne s'inquiète pas de l'autre vie et qui ne s'attache pas à la tradition religieuse de l'islam. [...]

Quand tu mourras, tout ce qui te restera c'est un linceul de trois coudées :

Où sera alors ta selle? où seront tes objets de harnachement?

Où seront les divertissements et les réunions galantes?

La sympathie est perceptible ici jusque dans l'invective; le sermonneur sait bien que lui-même et son interlocuteur s'accordent quant à ce qui a du prix. De ce 
dialogue, traversé de complicité, l'étranger à qui l'on fait la guerre est l'occasion mais en aucun cas le destinataire.

Il y a, en un mot, ceux à qui je fais la guerre en le leur disant, dans un dialogue poétique comparable à celui que j'entretiens avec mes contribules, et ceux dont je dis que je leur fais la guerre, dans des poèmes destinés à d'autres qu'eux. Pour les hommes qui ont composé ces poèmes, l'étranger commence là où l'échange poétique cesse de se superposer à l'échange guerrier. La où la guerre intestine voit le tumulte des armes s'accompagner de la rumeur des poèmes échangés, la guerre étrangère met face à face des hommes se combattant, si l'on peut dire, en silence. La transition de l'une à l'autre n'est pas brutale. À leurs voisins immédiats, avec qui ils partagent au moins la religion et qui sont parfois leurs alliés contre d'autres Touaregs, ils adressent encore la parole quand ils leur font la guerre, mais dans des apostrophes qui restent impersonnelles et dont ils ne semblent guère se soucier qu'elles leur parviennent vraiment. Avec les Français, dont le genre de vie est tout autre et qui sont à leurs yeux des Infidèles, ils n'ont aucune parole à échanger. C'est cependant grâce à l'un de ces étrangers, mort lui-même au cours d'une guerre où une grande partie des Touaregs s'unit contre les Infidèles, que nous sont parvenus les poèmes où nous retrouvons aujourd'hui en filigrane le tracé de cette frontière de silence. 


\section{Bibliographie}

ALBAKA, M. et CASAJUS, D.

1992 Poésies et chants touaregs de l'Ayr, Paris, L'Harmattan.

ALOJALY, Gh

1975 Histoire des Kel Denneg. Copenhague, Akademisk Forlag.

BOURGEOT, A.

1995 Les sociétés touarègues. Nomadisme, identité, résistances, Paris, Karthala.

CASAJUS, D.

1989 «Le poète et le silence » in Graines de parole, Écrits pour Geneviève Calame-Griaule, Paris, Éditions du CNRS.

1995 «Les amis français de la "cause touarègue" », Cahiers d'études africaines, 137, XXXV-1 : 237-250.

DUVEYRIER, $\mathrm{H}$.

1864 Les Touaregs du Nord, Paris, Challamel aîné.

FOUCAULD, C. de

1925-1930 Poésies touarègues (dialecte de l'Ahaggar), Paris, Leroux, 2 tomes.

1954 Lettres inédites au général Laperrine pacificateur du Sahara, Paris, La Colombe.

FOUCAULD, C. et A. de CASSALANTI-MOTYLINSKI

1984 Textes touaregs en prose, Aix-en-Provence, Édisud.

GALAND, L.

1980 «Le rezzou dans la poésie traditionnelle de l'Ahaggar», Atti della settimana internationale di studi mediterranei medioevali et moderni Cagliari, 27 aprile- $1^{\circ}$ maggio 1979, Milan, Giuffrè : 99-111.

1981 «Le campement dans la poésie traditionnelle de l'Ahaggar», Itinérances, II : 53-60.

1986 «Le vol de bétail dans le monde berbère et le monde méditerranéen », Atti del Congresso Internazionale di Amalfi, 5-8 dicembre 1983, Napoli, Istituto Universitario Orientale : 369-378.

GALAND-PERNET, P. 
1978 «Images et image de la femme dans les poésies traditionnelles de l'Ahaggar », Bulletin de littérature orale arabo-berbère 9 :5-52.

1984 «Le thème de l'errance dans les littératures berbères », Itinéraires, Littératures et contacts de culture, $4-5$ : 269-310.

GARDEL, G.

1961 Les Touaregs Ajjer, Alger, Baconnier.

HANOTEAU, A.

1896 Essai de grammaire de la langue tamachek, Alger, Carbonnel.

\section{KERGOAT, L.}

1988 Charles de Foucauld et l'Islam. Politique et mystique, Thèse d'État, Paris-Sorbonne, 2 t.

\section{NICOLAISEN, J.}

1963 Culture and Ecology of the Pastoral Tuareg, Copenhague, The National Museum of Copenhagen.

PRASSE K. G.

1990 Introduction, in, Mokhamed, G. et K.-G. Prasse, Poèmes touaregs de l'Ayr, Copenhague, Museum Tusculanum Press, Tome 2.

TRIAUD, J.-L.

1995 La légende noire de la Sanûssiya. Une confrérie musulmane saharienne dans le regard français (1840-1930), Paris, Éditions de la Maison des sciences de l'homme, 2 tomes.

VERNANT, J.-P.

1968 Introduction, in J.-P. Vernant (éd.), Problèmes de la guerre en Grèce ancienne, Paris-La Haye, Mouton, 1968 : 9-30. 
* Une version de ce texte, abrégée et adaptée pour un public plus large, est l'un des paragraphes de l'introduction d'une réédition des Poésies touarègues de Charles de Foucauld à paraître chez Albin Michel.

${ }^{1}$. Pour une période un peu postérieure, nous disposons de la correspondance des acteurs de l'insurrection senoussistes. Mais, comme J.-L. Triaud le relève, ces traces écrites ne sont guère nombreuses et les originaux sont souvent perdus (Triaud, 1995, II : 851, note 2).

2. Le terme, noté avec la transcription de C. de Foucauld comme tous les termes vernaculaires, désigne ici un chef de tribu noble qui ne reconnaît la suzeraineté d'aucun autre.

${ }^{3} .28$ : 13-15 (I, 42). On renvoie au numéro du poème et aux vers, puis au numéro du tome et à la page de Foucauld, 1925-1930.

${ }^{4}$. On a vu que des négociations avaient eu lieu devant Ghât, avant que ne commence la première bataille de cette guerre. L'introduction du poème 97 (I, 175) fait également état de négociations lors d'une rencontre entre les Kel-Ähaggar et un rezzou ioullemmeden ; l'affrontement fut cette fois évité. Ces adversaires se parlaient donc, jusque sur le champ de bataille.

5. $56: 12-14(\mathrm{I}, 91-92)$.

${ }^{6} .260$ (I, 477 sqq.).

${ }^{7}$. Par exemple en $76: 1$ (I, 126) ; $317: 5$ (I, 588) ; $540: 1-3$ (II, 380).

${ }^{8}$. Par exemple en $133: 9$ (I, 230) ; $225: 14-16$ (I, 417).

${ }^{9} .453: 14-16$ (II, 173). C'est aussi le cas au vers 2 du poème 444 (II, 136), et au vers 39 du poème 443 (II, 134). Mais dans le second cas l'auteur se trompe ; il a pris celui qu'il a tué pour un autre.

${ }^{10} .75: 23$ (I, 124).

${ }^{11} .52: 1-3$ (I, 77-78). Pour les échanges successifs du dialogue qui suit, voir : 527 : 1, 4-5 (II, 360-361) ; 53 : 27-28 (I, 84-85) ; 529 : 1-4 (II, 362-363) ; 54 : 1-3 (I, 86). Elghâlem et Äkhenna ont échangé des poèmes en d'autres occasions : 51 (I, 75-77) et 526 (II, 358-359).

12. 539 : 1-5 (II, 378-379), et $134: 1-5$ (I, 232), pour la réplique d'Éberzeouêl. Les auteurs des poèmes 55 (I, 86-89) et 124 (I, 215-217), composés à la même époque, s'emploient eux aussi à discréditer le parti de la paix, mais il s'adressent à leur propre camp. 
${ }^{13} .132: 6-8$ (I, 227-228), et $360: 5-7$ (II, 10) pour la réplique de Sîdi.

14. Le fait probable que le poème 265 (I, 497), composé en 1902, soit selon C. de Foucauld une imitation du poème d'Ähitaghel cité plus haut, composé en 1877, apporte un élément de preuve comparable. Ces remarques sur la fidélité de la conservation du texte des poèmes ne sont pas une digression. Si l'on tient que ces poèmes sont un témoignage sur ce que pensaient leurs auteurs, il importe en effet d'avoir sinon une preuve du moins des raisons solides de penser qu'ils sont conservés à peu près fidèlement depuis la date de leur composition.

${ }^{15}$. $405: 1-6$ (II, 68-69), et, pour les répliques : $168: 1-3$ et 8-10 (I, 287-288) ; 566 : 1-4 (II, 432).

${ }^{16}$. Sur ce violon, et sur l'expression « violon ! d dans l'extrait suivant, voir plus loin., p. 17 sqq.

17. Voir 28 : 7-11 (I, 41-42) ; $42: 5-9$ (I, 59-60) (l'auteur avoue sa propre lâcheté, ce qui est exceptionnel) ; $77: 42-51$ (I, 141-142) ; $443: 6-10,39-45$ (II, 129-135) ; $444: 1-3$ (II, 136) ; $451: 15-38,50-58$ (II, 156-162) ; 452 : 1-9, 18-21, 27-31, 36-41 (II, 164-170) ; $453: 6-39$ (II, 172-177) ; $454: 1-32$ (II, 179-183).

${ }^{18}$. Peut-être faut-il penser que la rumeur atteignait le camp adverse avant tout sous la forme, «officielle », de poèmes, alors qu'elle était nécessairement multiforme tant qu'on restait à l'intérieur du même camp.

${ }^{19} .332$ : 18-20 (I, 614). Voir aussi 256 (I, 473-474).

${ }^{20}$. L'invocation des femmes aimées fait partie des lois du genre. Citons, entre autres : $27: 1$ (I, 39) ; $28: 1-3$ (I, 40) ; $31: 1$-4 (I, 45) ; $56: 47-48$ (I, 97-98) ; $77: 55$ (I, 143) ; $97: 7-8$ (I, 177) ; $133: 3$ (I, 229) ; $172: 1$ (I, 294) ; $411: 1$ (II, 75) ; $453: 44$ 48 (II, 178) ; $531: 5-8$ (II, 365).

${ }^{21}$. 452: 27-29, 32-35 (II, 168-169). Voir aussi (outre les textes cités à la note précédente) : $28: 1-3$ (I, 40) ; $46: 1$-3 (I, 66) ; $371: 1-5$ (II, 25-26) ; $479: 1$ (II, 279). Dans le vers 11 du poème 511 (II, 328), c'est une poétesse qui demande à ses compagnes d'acclamer la bravoure d'un homme

${ }^{22} .262: 1-4(\mathrm{I}, 487-488)$

${ }^{23}$. Son nom apparaît également en $24: 1$ (I, 36) ; $42: 10$ (I, 60) ; $52: 4$ (I, 78) ; 77 : 52, 55 (I, 142-143) ; $364: 4$ (II, 18).

${ }^{24} .75: 22(\mathrm{I}, 123)$.

25. 75 : 24-26 (I, 124).

${ }^{26} .133: 8(\mathrm{I}, 230)$. 
27.499 : 9-10 (II, 305-306).

28. L'ähâl était une réunion galante où il était d'usage que les jeunes gens s'assemblent à la nuit tombée pour s'y divertir et y deviser.

${ }^{29} .535$ (II, 369 sqq.).

${ }^{30} .321: 3(\mathrm{I}, 594)$.

${ }^{31}$. Foucauld, 1925-1930, I : 82, dans la note du vers 20 du poème 53.

32. Ibid., II : 60. L'auteur précise cependant que la chose était considérée comme déshonorante.

${ }^{33} .113: 1-4$ (I, 199).

${ }^{34} .94$ : 5-7 (I, 168). Voir aussi les autres poèmes mentionnés par L. Galand.

$35.530: 1-5$ (II, 363-364).

${ }^{36}$. Sur les thèmes de la poésies élégiaque, le texte de référence est Galand-Pernet 1978 ; voir aussi Casajus, 1989 et Prasse, 1990.

${ }^{37} .225: 3,7-12$ (I, 415-417). Voir aussi : 77 : 1-4 (I, 133-134) ; $110: 1-7$ (I, 195196) ; $291: 6-8$ (I, 549-550) ; $317: 3-4$ (I, 587-588) ; 338 : 2-4 (I, 621-622) ; 478 : 17-18 (II, 274) ; 538 : 3-4 (II, 377-378). Ce thème se distingue parfois mal de la simple invocation des femmes aimées.

${ }^{38} .275$ : 9-10 (I, 515) ; 281 : 6-7 (I, 527). L. Galand (1980) cite également le poème 178.

${ }^{39}$. Foucauld, 1925-1930, I : 562, note du vers 2 du poème 301.

40. $191: 1-4$ (I, 326).

${ }^{41} .114: 1-5$ (I, 200-201) ; Voir aussi : $79: 5$ (I, 146) ; $132: 5$ (I, 227) ; $161: 10-11$ (I, 276) ;

$42.311: 6-7$ (I, 578).

43. 332 : 15-17 (I, 614); voir aussi 159: 1 (I, 269) ; $431: 5$ (II, 105), ce dernier composé à l'occasion d'une guerre étrangère.

${ }^{44} .168: 2$ (I, 287) ; $549: 1$ (II, 390).

${ }^{45}$. Voir l'introduction du poème 176 (I, 301).

${ }^{46} .75: 27-30$ (I, 124-125). 
${ }^{47}$. Le même auteur a consacré à ce violon un poème qui est un éloge métonymique de Tèhît. Voir 72 (I, 116-117).

${ }^{48} .451$ et 452 (II, 153-170).

${ }^{49} .308$ (I, 570-573) ; 309 (I, 573-576) ; 321 (I, 594-595) ; 322 (I, 595-596) ; 508 (II, 322-323). Voir aussi des allusions à des querelles d'adolescents dans les poèmes 314 (I, 584-585) ; 324 (I, 597-599) ; 325 (I, 600-601) ; 326 (I, 601-603) ; 327 (I, 604$605)$.

50. 574 (II, 445-447).

${ }^{51} .227$ (I, 427-428) ; 268 (I, 501-502) ; 270 (I, 503-505).

${ }^{52}$. Il sont regroupés sous le numéro 426 (II, 96-99).

${ }^{53} .117$ (I, 205-206) ; 118 (I, 206-208) ; 148 (I, 255-256) ; 155 (I, 263-264) ; 184 (I, 315-316) ; 208 (I, 348-349) ; 223 : 14 sqq. (I, 407-413) ; 333 (I, 615-617) ; 362 (II, 14-15) ; 422 (II, 86-87) ; 440 (II, 123-124). Les poèmes 445 (II, 138) et 574 (II, 445 447) sont composés, l'un contre une femme qui a dédaigné l'auteur, l'autre contre un rival heureux, sans qu'il soit question de mariage. Dans le poème 235 (I, 439-441), c'est une femme qui regrette le mariage d'un ami.

$54.222: 138,140-144$ (I, 403-404)

${ }^{55} .101$ (I, 183-185) et 108 (I, 192-193).

56. 157 (I, 266) ; 158 (I, 267-268) ; 202 (I, 341-342) ; 217 (I, 364-366) ; 231 (I, $432-$ 434) ; 238 (I, 446-447) ; 239 (I, 447-449) ; 259 (I, 476-477) ; 402 (II, 63-64) ; 407 (II, 69-70) ; 408 (II, 70-72) ; 448 (II, 144-145) ; 450 (II, 149-153) ; 473 (II, 258259) ; 512 (II, 328-329).

${ }^{57} .91$ (I, 163-164).

${ }^{58} .493$ (II, 294).

59.494 : 1-5 (II, 295).

${ }^{60} .391$ (II, 47-49) ; 467 (II, 247-248) ; 468 (II, 248-249). L'auteur du poème 239 (I, 447-449), accusée d'avoir deux amants, ne se justifie pas de cette accusation, mais se contente d'invectiver l'accusatrice. On pense à ces poèmes composés à propos d'un combat où il moins question de l'ennemi que des rivalités entre compagnons d'armes.

${ }^{61} .447$ (II, 140-143) ; 448 (II, 144-145) ; 533 (II, 366-367). Voir aussi 230 (I, 431432).

${ }^{62} .572: 1-5$ (II, 443-444). 
${ }^{63} .34$ (I, 50) ; 61 (I, 103-104) ; 69 (I, 112-114) ; 81 (I, 147-148) ; 84 (I, 151-152) ; 138 (I, 236-238) ; 139 (I, 238-239) ; 143 (I, 246-248) ; 149 (I, 256-258) ; 160 (I, 271-273) ; 171 (I, 293-294) ; 182 (I, 313-314) ; 185 (I, 317-319) ; 186 (I, 319-320) ; 190 (I, 324-325) ; 195 (I, 330-331) ; 240 (I, 449-442) ; 241 (I, 451-452) ; 248 (I, 462-463) ; 282 (I, 528-532) ; 319 (I, 590-591) ; 363 (II, 15-17), 373 (II, 27-28) ; 399 (II, 57-59) ; 402 (II, 63-64) ; 432 (II, 106-107) ; 501 : 1-4 (II, 308-308) ; 524 (II, 353-354) ; 552 (II, 395-396).

${ }^{64}$. C'est le cas des poèmes 483 (II, 280-281) et 504 (II, 312-313) d'une part, 205 (I, 346) et 548 (II, 389-390) d'autre part, dont les auteurs s'invectivent mutuellement. Voir aussi 192 (I, 326-327) ; 277 (I, 518-520) ; 298 (I, 559-560). Il arrive qu'on ait du mal à en percevoir les motifs de ces invectives. Ainsi un poème, où l'auteur dit de Tegheichat oult Ibdeqqen que «même les chiens de veulent pas d'elle »; voir 568: 4 (II : 437). Tegheichat avait recueilli et soigné un tirailleur arabe blessé lors du massacre de la mission Flatters. Louis Kergoat (1988, I : 84, note 4) semble considérer que là est la raison de cette insulte, mais il y aurait alors une erreur de date de la part de Foucauld. Le poème est daté par C. de Foucauld de 1880, et la mission Flatters a été massacrée en 1881.

65. 125 (I, 217- 219) ; 237 (I, 444-445) ; 285 (I, 536-538) ; 286 (I, 538-541) ; 470 (II, 250-252). On trouve aussi des plaisanteries dans les poèmes 198 (I, 334-335) et 379 (II, 33-34). Les poèmes 380 (II, 35) et 433 (II, 108-109) sont des plaisanteries un peu aigres échangées entre des hommes de deux tribus voisines, où un vers de l'attaque est repris mot pour mot dans la réponse.

${ }^{66} .315$ (I, 585-586) ; 316 (I, 586-587) ; 330 (I, 608-609) ; 331 (I, 609-611).

${ }^{67} .293(\mathrm{I}, 551-553)$.

${ }^{68}$. J'ai prolongé ici des remarques déjà amorcées dans un texte consacré à la rébellion touarègue au Niger (Casajus, 1995).

${ }^{69} .56(\mathrm{I}, 89-98)$.

${ }^{70} .46(\mathrm{I}, 65)$; introduction du poème 42 (I, 58) ; introduction du poème 159 (I, 268269) ; $226(\mathrm{I}, 418)$.

${ }^{71}$. $522: 3-7$ (II, 350). Voir aussi $511: 1-5$ (II, 327) ; $565: 1$-2 (II, 430) ; 569 (II, 437-438) ; 570 (II, 438-439). L'adresse est le plus souvent indirecte, comme dans le texte cité : on demande à des compagnons de transmettre ses menaces aux Arabes ; mais c'est là une figure de style présente aussi dans certains des poèmes inspirés par les guerres internes.

${ }^{72} .549: 15$ (II, 393).

${ }^{73} .159: 1$ (I, 269) ; $372: 1-5$ (II, 26-27) ; 544 (II, 384-386) ; 
${ }^{74} .145$ (I, 250-251) ; 146 (I, 252-253) ; $312: 12-18$ (I, 580-581).

${ }^{75} .19$ : 15 sqq. (I, 23) ; $46: 10-12$ (I, 67) ; $94 ; 19-24$ (I, 170-171) ; $159: 10$ (I, 271) ; $300: 1-2$ (I, 561) ; $312: 19-21$ (I, 581-582) ; 479 (II, 275-277) ; 523 : 1-5 (II, 351-352).

${ }^{76}$. Le poème 565 (II, 430-431) leur est certes adressé, mais c'est le seul sur les cinq poèmes consacrés à ce rezzou.

${ }^{77} .556: 10$ (II, 405).

${ }^{78} .57$ (I, 98-99) ; 163 (I, 277-278) ; 164 (I, 278-281) ; 166 (I, 284-286) ; 263 (I, 489494) ; 264 : 9-13 (I, 496-497) ; 265 : 1 (I, 497) ; 292 (I, 550-551).

${ }^{79} .265$ : 2 (I, 497) ; $507: 7$ (II, 317). Le mot que nous traduisons par païen est le mot d'origine arabe äkâfer, que les Touaregs utilisent aujourd'hui encore pour désigner les Occidentaux.

${ }^{80} .345$ : 11 (I, 631). Ce poème, très tardif, date de 1906. Il témoigne de la part de son auteur d'une acceptation de la situation coloniale qu'on ne trouve dans aucun autre poème du recueil, hormis ceux de Mokhammed äg Ghëli, dont le cas est très particulier.

${ }^{81} .251: 2$ (I, 466) ; $454: 27$ (II, 183) ; $462: 75$ (II, 222).

${ }^{82} .507$ : 18-25 (II, 319-320).

${ }^{83} .462$ : 75-78, 80-82 (II, 222-224). 